\title{
Waveguide $\mathrm{DC}$ breaks for $\mathrm{TE}_{01}$ and $\mathrm{HE}_{11}$ microwave transmission lines
}

\author{
D. I. Sobolev, A. P. Gashturi, G. G. Denisov, G. I. Kalynova, D. A. Lukovnikov \\ Institute of Applied Physics, Nizhny Novgorod, Russia, nrtnm@mail.ru
}

\begin{abstract}
Introduction
Oversized waveguide are widely used in high power and high frequency microwave setups. Low modes in such waveguides have relatively low losses in gaps several wavelengths long [1], which make it possible to use waveguide gaps for DC insulation. Further optimization of DC breaks is possible by introducing waveguide taper converters at both sides, which are designed to increase beam quality and increase waveguide radius at its ends. The method of synthesis of such converters developed earlier by authors allowed to decrease the losses by an order of magnitude or more in comparison to simple break [2], but improvements are possible to make the converters smaller and keep losses near the theoretical minimum limited by diffraction.
\end{abstract}

\section{Waveguide system synthesis with multi-region field analysis}

The optimized DC break consists of two waveguide converters separated with air or vacuum gap and some other components, which may be exposed to high-power electromagnetic field, e.g. insulated box around the gap and some parts of cooling system. Direct analysis of fields in the whole system may take quite a long time, and synthesis usually requires several tens of calculations. To increase the speed, we separated the calculations into several parts (Fig. 1). Fields in region II can be calculated by fast methods like coupled modes or mode-matching, but region III of arbitrary shape requires slow direct methods. In low-loss DC break, only a few low modes are present inside the waveguide converters, therefore we can calculate the radiation of each of these mode from transmitting waveguide cross-section into region III once and then decompose the results into several waveguide modes at the receiving end to form a lowdimension transmission matrix. In general case, this approach has very low precision, but in a special case where transmission efficiency is very high, it produces good results and allows us to skip slow direct field calculations within the iteration loop.

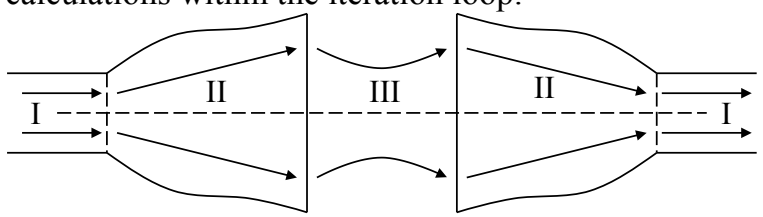

Fig. 1. Waveguide break parts: I - regular waveguides of transmission line, II - waveguide converters, III - interior of insulated box.

The synthesis of converters is based on the previous work made by authors [3]. At the beginning of every iteration step the system (Fig. 2) consists of the given input cross-section $S_{1}$, given output cross- section $S_{2}$ and initial conducting wall surface $S_{0}$. If we consider only one orientation, then conversion efficiency can be is increased after iteration by calculating two field distributions inside the waveguide. The first one is calculated using the given $\mathbf{E}_{1}$ and $\mathbf{H}_{1}$ fields at the input as a boundary condition, the second one by using the spatially reversed desired $\mathbf{E}_{2}$ and $\mathbf{H}_{2}$ fields at the output. Small and smooth deformation $l$ orthogonal to waveguide wall affects the efficiency (mutual power) $P$ according to formulas

$$
\Delta P=\int_{S_{0}} l F d S, \quad F=-i k\left(\mathbf{H}_{\tau 1} \mathbf{H}_{\tau-2}+\mathbf{E}_{n 1} \mathbf{E}_{n-2}\right)
$$

where $\tau$ and $n$ means tangential and normal field components, respectively. We can minimize the average square of deformation (i.e. norm) using real and imaginary parts of (1) as equality constraints with given $\Delta P$, and in this simple case the deformation takes the form

$$
l=\alpha \operatorname{Re} F+\beta \operatorname{Im} F
$$

where constants $\alpha$ and $\beta$ are expressed by $\Delta P$ and surface integrals of function $F$ [4].

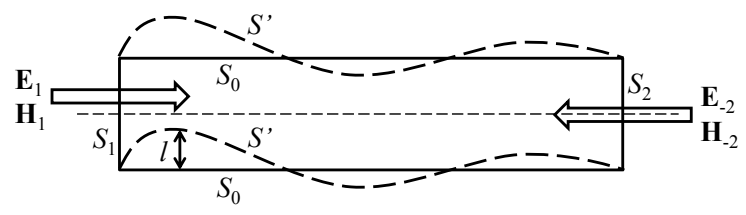

Fig. 2. Waveguide synthesis notations: two given waveguide cross-sections $S_{1}$ and $S_{2}$ (input and output, respectively), the waveguide surface before iteration $S_{0}$ and the surface after iteration $S^{\prime}$, the deformation value $l$ at some point of the surface, the specified input fields $\mathbf{E}_{1}$ and $\mathbf{H}_{1}$, and the reversed desired output fields $\mathbf{E}_{-2}$ and $\mathbf{H}_{-2}$.

At the first iteration the fields in the simplified representation are far from the real ones, but as the synthesis converges the accuracy increases.

\section{DC break for $\mathrm{TE}_{01}$ mode}

The break for $\mathrm{TE}_{01}$ transmission line have to be able to withstand $80 \mathrm{kV}$ voltage, the operating frequency of the setup is $28 \mathrm{GHz}$, and the radius of the transmission line is $16.27 \mathrm{~mm}$. The gap length equal to $100 \mathrm{~mm}$ is enough withstand the required voltage with allowed static field strength $30 \mathrm{kV} / \mathrm{cm}$, as the field bigger near the waveguide ends. The waveguide radius at its ends have to be $50 \mathrm{~mm}$ to transmit the wave beam with very low losses. The length of each synthesized waveguide converter is $318 \mathrm{~mm}$, and the total calculated efficiency is $99.8 \%$ at the central frequency (Fig. 3). The DC break passed the tests at voltages up to $85 \mathrm{kV}$ limited by the voltage source available, and low power microwave tests which show the transmission efficiency $99 \% \pm 1 \%$. 

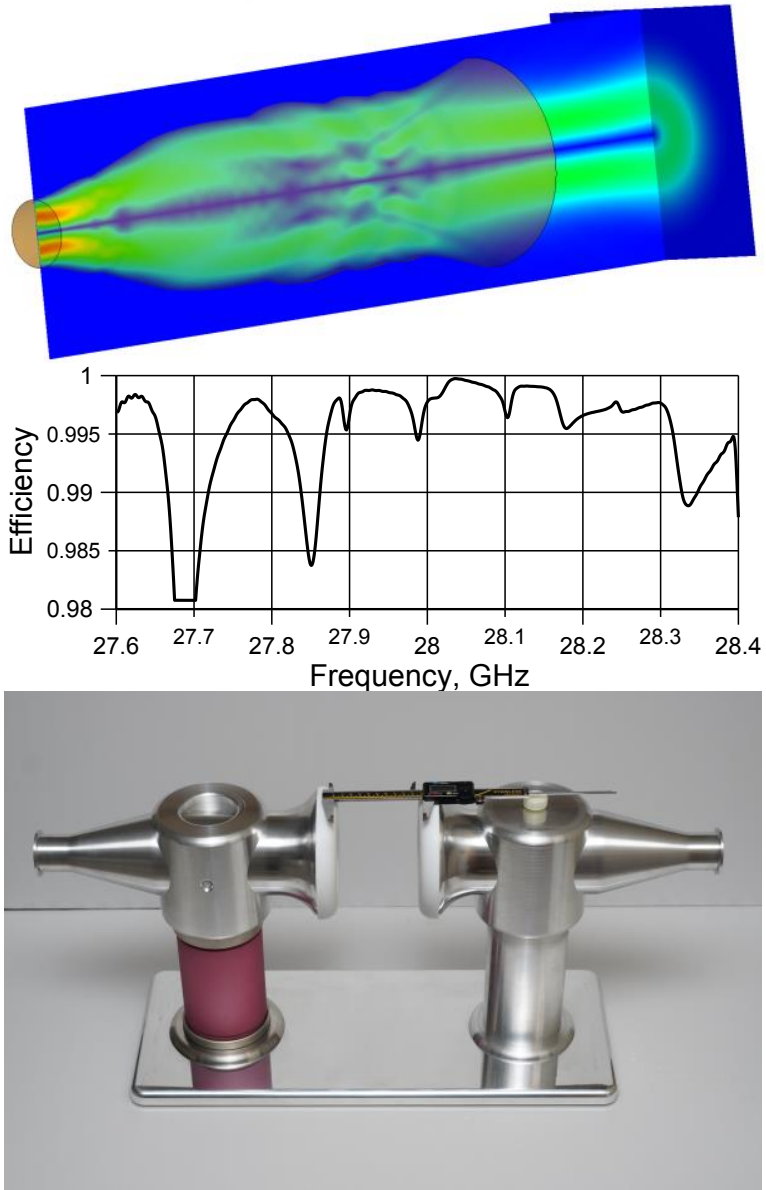

Fig. 3. Top - waveguide converter for $\mathrm{TE}_{01}$ mode break and cross-section of the electric field; middle - calculated transmission efficiency (FDTD); bottom - DC break in configuration used for high voltage tests.

\section{Wideband DC break for $\mathrm{HE}_{11}$ mode}

Special wideband version of DC break was synthesized and tested for $\mathrm{HE}_{11}$ mode. Able to withstand
$50 \mathrm{kV}$, this break has waveguide converters $270 \mathrm{~mm}$ length each with radius changing from $20 \mathrm{~mm}$ to $31.75 \mathrm{~mm}$, gap length $63.5 \mathrm{~mm}$ and was designed to work in frequency band 28.5-31.5 GHz. Calculated efficiency is at least $97.5 \%$ in $5 \%$ frequency band and at least $95 \%$ in $10 \%$ frequency band. Measured efficiency agrees well with calculations in $5 \%$ band, but drops in $10 \%$ band (Fig. 4), which may be the result of the limited precision of low-dimension transmission matrix.

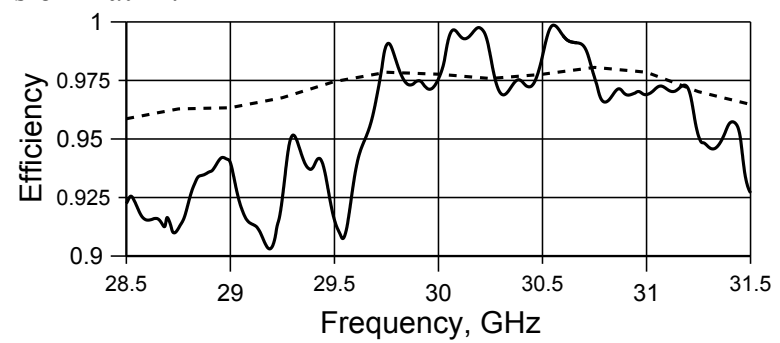

Fig. 4. Wideband DC break for $H_{11}$ mode: dashed line calculated transmission efficiency, solid line - measured transmission efficiency.

\section{References}

1. Wagner, D., Thumm, M., Kasparek, W., Miiller, G. $A$., and Braz, O. Prediction of TE-, TM-, and hybrid mode transmission losses in gaps of oversized waveguides using a scattering matrix code // Int. J. Infrared and Millimeter Waves. 1996. V. 17. No. 6. P. 1071-1081.

2. Sobolev, D.I., Chirkov, A.V., Denisov, G.G., Lukovnikov, D.A., Malygin, V.I. Minimization of diffraction losses in big gaps of multi-mode waveguides // Int. J. Infrared and Millimeter Waves. 2005. V. 26. No. 7. P. 953-966.

3. Sobolev, D. I., Denisov, G. G. Principles of Synthesis of Multimode Waveguide Units // IEEE Trans. Plasma Sci. 2010. V. 38. No. 10. P. $2825-2830$. 